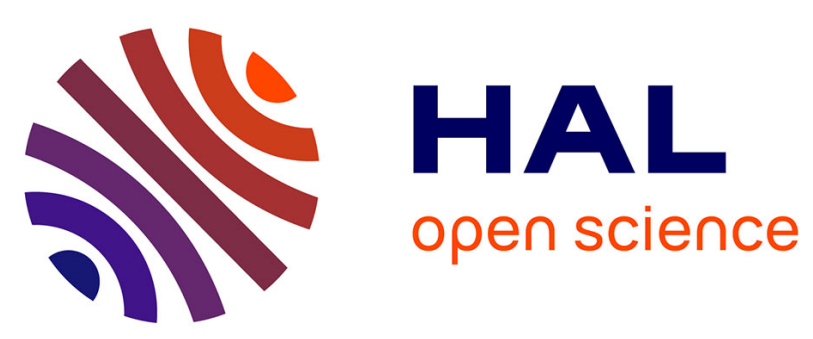

\title{
HYBRID MODEL OF ERYTHROPOIESIS AND LEUKEMIA TREATMENT WITH CYTOSINE ARABINOSIDE
}

Polina Kurbatova, Samuel Bernard, Nikolai Bessonov, Fabien Crauste, Ivan Demin, Charles Dumontet, Stephan Fischer, Vitaly Volpert

\section{To cite this version:}

Polina Kurbatova, Samuel Bernard, Nikolai Bessonov, Fabien Crauste, Ivan Demin, et al.. HYBRID MODEL OF ERYTHROPOIESIS AND LEUKEMIA TREATMENT WITH CYTOSINE ARABINOSIDE. SIAM Journal on Applied Mathematics, 2011, 71 (6), pp.2246-2268. 10.1137/100815517. hal-00538496v2

\author{
HAL Id: hal-00538496 \\ https://hal.science/hal-00538496v2
}

Submitted on 13 Nov 2012

HAL is a multi-disciplinary open access archive for the deposit and dissemination of scientific research documents, whether they are published or not. The documents may come from teaching and research institutions in France or abroad, or from public or private research centers.
L'archive ouverte pluridisciplinaire HAL, est destinée au dépôt et à la diffusion de documents scientifiques de niveau recherche, publiés ou non, émanant des établissements d'enseignement et de recherche français ou étrangers, des laboratoires publics ou privés. 


\title{
HYBRID MODEL OF ERYTHROPOIESIS AND LEUKEMIA TREATMENT WITH CYTOSINE ARABINOSIDE
}

\author{
POLINA KURBATOVA*, SAMUEL BERNARD ${ }^{\dagger}$, NIKOLAI BESSONOV ${ }^{\ddagger}$, FABIEN

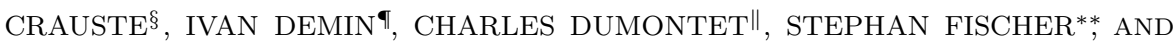 \\ VITALY VOLPERT ${ }^{\dagger \dagger}$
}

\begin{abstract}
A hybrid model of cell population dynamics, where cells are discrete elements whose dynamics depend on continuous intracellular and extracellular processes, is developed to simulate the evolution of immature red blood cells in the bone marrow. Cell differentiation, self-renewal or apoptosis are determined by an intracellular network, based on two proteins, Erk and Fas, and described by ordinary differential equations, and by local extracellular regulation performed by Fasligand, a protein produced by mature cells whose concentration evolution is represented by a partial differential equation. The model is used to study normal and leukemic red blood cell production (erythropoiesis), and treatment of leukemia. Normal cells are assumed to have a circadian rhythm that influences their cell cycle progression, whereas leukemic cells, are assumed to escape circadian rhythms. We consider a treatment based on periodic administration of Ara-C, an anti-cancer agent targeting cells in DNA synthesis. A detailed pharmacodynamic/pharmacokinetic model of Ara-C is proposed and used to simulate the treatment. Influence of the period of the treatment and the day delivery time on the outcome of the treatment is investigated and stress the relevance of considering chronotherapeutic treatments to treat leukemia.
\end{abstract}

Key words. Hybrid model, leukemia treatment, chronotherapy, regulatory networks, cell cycle

AMS subject classifications. 92C30, 92C37, 92C50, 70F40, 68U20, 35Q70, 35Q92

\section{Introduction.}

1.1. Regulation of normal erythropoiesis. Erythropoiesis is a part of hematopoiesis, the process regulating blood cell production, and is responsible for red blood cell formation. Erythropoiesis occurs in discrete anatomic units of bone marrow, the erythroblastic islands. Erythroblastic islands consist of inner layers of erythroid progenitors and outer layers of erythroblasts organized around one or two macrophages, which promote erythroblast proliferation [11]. Erythroid progenitors, immature blood cells with abilities to proliferate and differentiate, divide to produce erythroblasts (mature progenitors), then reticulocytes (precursor cells) and finally mature erythrocytes (red blood cells), which leave the bone marrow and enter the blood flow.

Erythropoiesis is a robust system, able to keep and quickly restore an optimal number of circulating red blood cells even after pertubations e.g. after an injury. Inter-

${ }^{*}$ Université de Lyon ; Université Lyon 1 ; CNRS, UMR5208, Institut Camille Jordan; INSA de Lyon, F-69621 ; Ecole Centrale de Lyon ; 43 blvd du 11 novembre 1918, F-69622 Villeurbanne-Cedex, France (kurbatova@math.univ-lyon1.fr).

†Université de Lyon ; Université Lyon 1 ; CNRS, UMR5208, Institut Camille Jordan; INSA de Lyon, F-69621 ; Ecole Centrale de Lyon ; 43 blvd du 11 novembre 1918, F-69622 Villeurbanne-Cedex, France (bernard@math.univ-lyon1.fr).

¥Institute of Mechanical Engineering Problems, 199178 Saint Petersburg, Russia

$\S$ Université de Lyon ; Université Lyon 1 ; CNRS, UMR5208, Institut Camille Jordan; INSA de Lyon, F-69621 ; Ecole Centrale de Lyon ; 43 blvd du 11 novembre 1918, F-69622 Villeurbanne-Cedex, France (crauste@math.univ-lyon1.fr).

INovartis Pharma AG, Department of Modeling and Simulation, Basel, Switzerland

"Unité INSERM U590, University Lyon 1, 8 avenue Rockefeller 69008 Lyon

** Université de Lyon, CNRS, INRIA ; INSA-Lyon, LIRIS Combining, UMR5205, F-69621, France

${ }^{\dagger}$ Université de Lyon; Université Lyon 1 ; CNRS, UMR5208, Institut Camille Jordan; INSA de Lyon, F-69621 ; Ecole Centrale de Lyon ; 43 blvd du 11 novembre 1918, F-69622 Villeurbanne-Cedex, France (volpert@math.univ-lyon1.fr). 
nal and external regulations form complex positive and negative regulation pathways that maintain the integrity of the erythropoietic system $[14,15,16,20]$. The hormone erythropoietin (Epo) acts on erythroid progenitors by promoting proliferation and cell differentiation, and by protecting cells from apoptosis [28]. Its anti-apoptotic effect is balanced by cell-cell interaction, during which binding of reticulocyte-produced Fas-ligand to the membrane protein Fas stimulate apoptosis of immature erythroid progenitors [19]. Fas also induces differentiation, while Erk protein kinase induces erythroid progenitor self-renewal.

1.2. Treatment of acute myeloid leukemia with Ara-C. Leukaemia is a malignant disease characterized by abnormal proliferation of immature blood cells or haematopoietic stem cells within the bonne marrow. There are four types of leukemia: myelogenous and lymphocytic (according to the haematopoietic lineage involved in the disease), each of which being acute (rapid increase of immature blood cells) or chronic (excessive production of relatively mature blood cells). Erythroleukemia is one of the the eight subtypes of acute myeloid leukemia (AML). It was first described by Di Guglielmo in the early twentieth century [30], it is characterized by a proliferation of erythroid and myeloid (white cells) progenitors, and sometimes a pure erythroid proliferation is observed [27]. A dysfunction of the Epo-mediated apoptosis has been stressed to be a causative agent of erythroleukemia. Self-activation of Epo-receptors at the surface of erythroid progenitors [18] could explain the sudden proliferation of immature erythroid cells. Yet, experiments conducted by [29] indicate that autocrine stimulation by erythropoietin in mice could result in intense proliferation of erythroid progenitors but never triggered erythroleukemia. Self-activation of Eporeceptors cannot explain the appearance of erythroleukemia without destabilisation of other signaling pathways.

During the past decade the first line therapy for AML patients has been daunorubicin or idarubicin in combination with cytosine arabinoside (Ara-C), although new investigational options exist [23]. Ara-C is characterized by a short half-life and targets cells during DNA synthesis (S-phase of the cell cycle). After intravenous administration, the drug is rapidly metabolized, by deamination in the liver and kidney, to its inactive form uracil arabinoside (Ara-U). When in the bone marrow, it penetrates proliferating cells membrane and it can be transformed into its active form arabinoside triphosphate (Ara-CTP), which participates in the DNA duplication, replacing natural nucleotides. When the proportion of Ara-CTP in the DNA becomes sufficiently high, the cell dies by apoptosis.

Ara-C acts on all proliferating cells whether they are leukemic or normal. Therefore, the aim in optimizing drug administration schedule is to increase cytotoxicity for leukemic cells and tolerance for normal cells. One possible approach to this problem is based on chronotherapy, in which drug administration is varied in time (chronomodulated) to exploit the small differences in the temporal organisation of the cell cycle between normal and leukemic cells.

Erythroid progenitors show specific daily variation in their DNA synthesis activity. 24 hours studies of healthy bone marrow cells showed circadian (about $24 \mathrm{~h}$ ) rhythms in proliferative activity [38]. On an average, the percentage of total bone marrow cells in DNA synthesis phase is greater at midday than at midnight. Myeloid and erythroid precursor cells display a daily peak in S-phase at 1 p.m. [39]. In contrast, leukemic cells display reduced rhythmicity, or even be arrhythmic [40]. This difference between healthy and leukemic cells allows could be exploited to reach a maximal efficacy and minimal toxicity by treating patients at specific times of the 
day. This strategy, termed chronotherapy, aims at decreasing toxicity and improving efficacy of the treatment by synchronizing drug delivery with biological rhythms $[1,32]$.

We present here a hybrid model with off-lattice cell dynamics that describes erythropoiesis to explore the interplay between extracellular and intracellular regulation for the normal and leukemic red blood cell formation. The model is hybrid in the sense that cells are modeled as discrete objects, while the extracellular space is continuous. We take a particular care in describing the erythroblastic island, which is thought to be important for erythropoiesis in vivo, and the effect of cell-cell interaction on cell fate decision. In the hybrid model, cell fate emerges from the intracellular state of each individual cells. This allows a much finer and more complex regulation, closer to the biology, and takes into account the heterogeneous and stochastic nature of cell fate. In contrast to standard macroscopic population models, the rates of selfrenewal, differentiation and apoptosis are not imposed by the model, but are obtained as a result of intracellular events that are specific to erythropoiesis. In Section 2, we introduce the hybrid model. In Section 3, we use the hybrid model to simulate the dynamics of normal and leukemic erythroblastic islands to find out improved administration schedules of the cell cycle phase-specific drug Ara-C. We also compare results of simulations with clinical data on treatment of leukemic haematopoiesis. In Section 4 , we discuss biological and computational issues.

\section{Mathematical model and methods.}

2.1. Hybrid models. We present in this section a hybrid model of erythropoiesis with off-lattice cell dynamics [5, 6, 7, 8, 9, 10, 22, 34]. In this approach, cells are modelled as discrete objects in the two-dimensional space. They interact with each other and with the surrounding medium mechanically and biochemically [17]. Cells have the ability to move, grow, divide, differentiate and die by apoptosis. The fate of each cell is determined by intracellular and extracellular regulatory networks. Intracellular regulatory networks are described by ordinary differential equations:

$$
\frac{d u_{i}(t)}{d t}=F\left(u_{i}(t), u\left(x_{i}, t\right)\right),
$$

where $u_{i}$ is a vector of intracellular concentrations of cell $i, u$ is a vector of extracellular concentrations, $F$ is a vector of reaction rates which is specified for each particular application. Intracellular concentrations $u_{i}$ are supposed to be uniformly distributed inside the $i$ th cell. Therefore these functions depend on time $t$ and they are independent of the space variable $x$. Values of intracellular concentrations can be different in different cells. At the same time, they can be influenced by extracellular concentrations $u(x, t)$. We will see below that cell motion will be reduced to motion of cell centers. The value of the extracellular concentration in the right-hand side of equation (2.1) is taken at the center $x_{i}$ of the $i$ th cell (see also Figure 2.1).

Extracellular concentrations [35] are described by reaction-diffusion equations:

$$
\frac{\partial u}{\partial t}=D \Delta u+G(u, \rho)
$$

The reaction rate $G$ is the rate of consumption or release of different species by cells. The reaction rate $G$ depends on the local cell density $\rho, D$ is the extracellular diffusion rate. The numerical resolution of this equation is based on a conventional implicit finite-difference alternative direction method. A specific feature of this model is that 


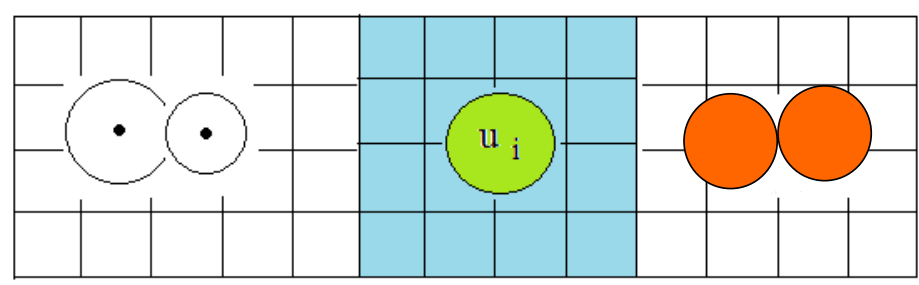

FIG. 2.1. Schematic representation of the hybrid model. The square grid shows the numerical mesh to solve reaction-diffusion equations (2.2). Cell centers move according to equations (2.3). The force $f\left(d_{i j}\right)$ between two cells depends on the distance between their centers (left). Intracellular concentrations $u_{i}(t)$ inside the ith cell are described by equations (2.1). They can be influenced by the extracellular concentrations $u(x, t)$ shown schematically as a blue field (center). If a cell dies by apoptosis, it is removed from the computational domain. If it divides, it increases its area. Cells grow by doubling their radii before dividing into two cells with a small radius (right).

cells are not necessarily small in comparison with the space step of the numerical mesh. A local cell concentration $\rho$ is computed as the total cell area inside each square of the grid.

The mechanical aspects of the model are based on previous work [8,9], and we briefly introduce them here. We model cells as elastic balls (compressible) with an incompressible core. Cell motion is determined by the sum of the mechanical forces acting on that cell from other cells. Under the assumption of small deformations, we can express the mechanical contact force acting between them as a function of the distance between their centers (Figure 2.1 (left)). The force between two particles centered at $x_{i}$ and $x_{j}$ is given by a function $f\left(d_{i j}\right)$ of the distance $d_{i j}$ between the centers. Cell motion is described by the displacement of its center by Newton's second law:

$$
m \ddot{x}_{i}+\mu m \dot{x}_{i}-\sum_{j \neq i} f\left(d_{i j}\right)=0,
$$

where $m$ is the mass of the cell, the second term in left-hand side describes the friction due to viscosity of the surrounding medium, the third term is the potential force between cells. The force between two spherical particles is

$$
f\left(d_{i j}\right)= \begin{cases}+\infty, & d_{i j}<d_{0}-2 H_{1} \\ K \frac{d_{0}-d_{i j}}{d_{i j}-d_{0}+2 H_{1}}, & d_{i j}<d_{0}, \\ 0, & d_{i j} \geq d_{0}\end{cases}
$$

where $d_{0}$ is the sum of cell radii, $K$ is a positive parameter, and $H_{1}$ is the size of the outer shell that form the compressible part of the cell. The force between the particles tends to infinity when $d_{i j}$ decreases to $d_{0}-2 H_{1}$. The force is repulsive if $d_{i j} \geq d_{0}$ and is null otherwise.

Intracellular concentrations determine how cells divide, differentiate or die. Particular cell fate is triggered when the intracellular concentration of some protein exceeds a threshold. The threshold represents the degree of activation required to trigger an irreversible cellular response downstream of the regulatory network modelled. In the numerical model, if a cell dies by apoptosis, it is removed from the computational domain. In order for a cell to divide, it increases its radius while preserving 
a circular shape during its growth. Here, radius doubles before division (Figure 2.1 (right)). More realistic cell shape can be considered, by taking a $\infty$-shape for instance $[6,7,8,9]$.

Parameter values of hybrid model are presented in Table 2.1 and valid for all numerical simulations and all cell populations unless explicitly stated.

2.2. Intracellular and extracellular regulation of normal erythropoiesis. In this section, we set up and calibrate the hybrid mode described above to the process of erythropoiesis. We consider two types of cells, erythroid progenitors and reticulocytes (Figure 2.2). Reticulocytes produce Fas-ligand that diffuses into the extracellular matrix and bind to Fas located on the membrane of erythroid progenitors. Erythroid progenitors also have Epo receptors (EpoR). Epo production depends on the number of erythrocytes in blood.

Intracellular regulation. We focus our attention on two key intracellular proteins, Erk and Fas, which are involved in cell fate decision. High Erk levels induce cell self-renewal, whereas high levels of Fas induce either cell differentiation or apoptosis. Evolution of the concentrations of these proteins inside an erythroid progenitor is described by a system of two ordinary differential equations presented in [16],

$$
\left\{\begin{array}{l}
\frac{d E_{i}(t)}{d t}=\left(\alpha(E p o)+\beta E_{i}(t)^{k}\right)\left(1-E_{i}(t)\right)-a E_{i}(t)-b E_{i}(t) F_{i}(t), \\
\frac{d F_{i}(t)}{d t}=\gamma(\text { FasL })\left(1-F_{i}(t)\right)-c E_{i}(t) F_{i}(t)-d F_{i}(t)
\end{array}\right.
$$

where $E_{i}(t)$ and $F_{i}(t)$ are the concentrations of active forms of Erk and Fas, respectively, in cell $i$ (cell numbering is determined by the discrete off-lattice model). Intracellular concentrations $E_{i}(\mathrm{t})$ and $F_{i}(t)$ are assumed to be uniform inside cell $i$. The right-hand sides of equations (2.5) depend on extracellular concentration of Fas-ligand, denoted FasL, and erythropoietin, denoted Epo, at the position of cell $i$.

Erk activation rate is proportional to the concentration of inactive Erk, $(1-$ $E_{i}$ ), where total Erk concentration has been normalized. Erk is activated by two pathways. Erk activation is self-amplifying through a positive feedback loop formed by the activation cascade Erk $\rightarrow$ Raf- $1 \rightarrow$ Mek $\rightarrow$ Erk (Figure 2.3). This positive loop is taken into account by the term $E_{i}^{k}$, with a cooperativity coefficient $k=2$. Erk is also activated by Epo, through the function $\alpha$. In a similar way, Fas activation by Fas-ligand is represented by the function $\gamma$. Erk/Fas activity are mutually exclusive. Active Erk inhibits Fas expression, and Fas activates the Ask-1/JNK/p38 pathway, which, in turn, inhibits Erk activation (Figure 2.3). Inhibition terms are modelled as proportional to $E_{i} F_{i}$. Finally, both Erk and Fas proteins are deactivated with rate constants $a$ and $d$.

The rate $\gamma$ is a function of FasL, with $\gamma(F a s L)=\gamma_{0}+k_{\gamma} F a s L$. This approximation is valid when Fas-ligand is low enough not saturate Fas receptors. Extracellular Epo concentration is physiologically regulated by oxygen levels in circulation. Here, as we concentrate on modelling the local regulation of erythropoesis, Epo level is assumed constant and uniformly distributed in the extracellular medium. Thus, the rate $\alpha$ in (2.5) will be considered as a positive parameter.

A detailed analysis of this system (2.5) is presented in [16, 20], and we briefly describe its dynamics. System (2.5) has between one and three stationary points [16], depending on parameter values. Conceptually, different steady states would correspond to different cell fates. This idea was exploited here and we modelled the Erk-Fas interaction as a bistable switch. Parameter values were chosen in such a way 


\section{Bone marrow}

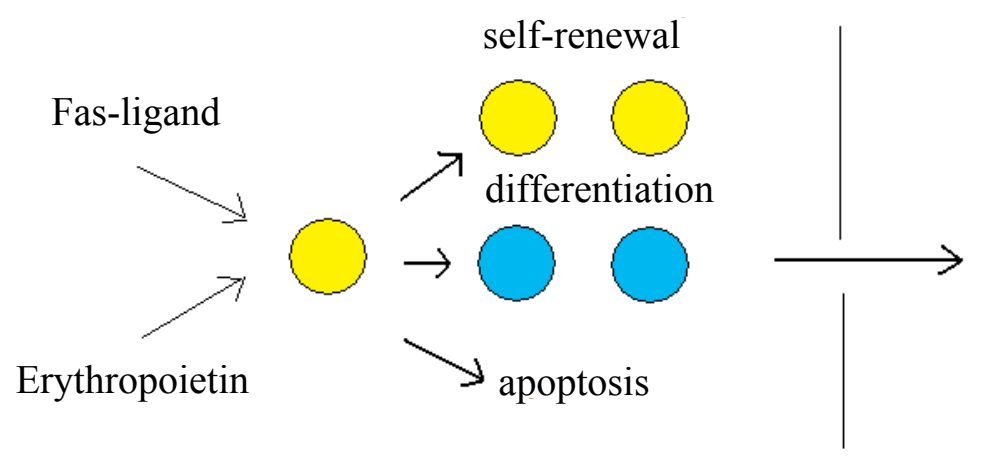

FIG. 2.2. Schematic representation of erythropoiesis. Erythroid progenitors (yellow cells) can self-renew producing two similar cells, differentiate producing two reticulocytes (blue cells) or die by apoptosis. Reticulocytes produce Fas-ligand which can promote differentiation and apoptosis of progenitors. Reticulocytes leave the bone marrow into blood flow where they become mature erythrocytes. Hormone erythropoietin, whose production rate depends on the number of erythrocytes in blood, increases the rate of self-renewal of progenitors and decreases their apoptosis.

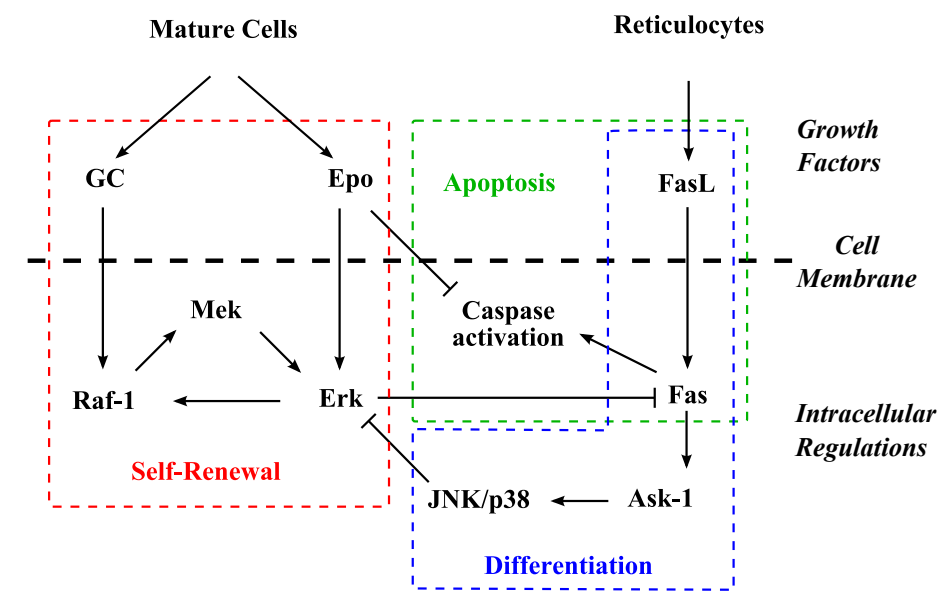

Fig. 2.3. Summary of intracellular protein regulatory network that determines erythroid progenitor fate [adapted from Crauste et al. [16], after Rubiolo et al. [37]].

that the system has three steady states, so as to give approximately correct proportion between self-renewing, differentiating and apoptotic cells and the correct response of the system to stimulation by the hormones. Values are given in Table 2.1. Bistability is illustrated in Figure 2.4, where points $A$ and $C$ are stable nodes, and point $B$ is an unstable saddle. Point $A$ corresponds to high levels of activated Fas and low levels of activated Erk, whereas point $C$ corresponds to low levels of activated Fas and high levels of activated Erk. When Erk-Fas concentration approaches point $A$ (high Fas, low Erk), the cell dies by apoptosis. When Erk-Fas concentration approaches point $C$ (low Fas, high Erk), the cell divides into two identical daughter cells. If the cell does not self-renew or die, it differentiate. 


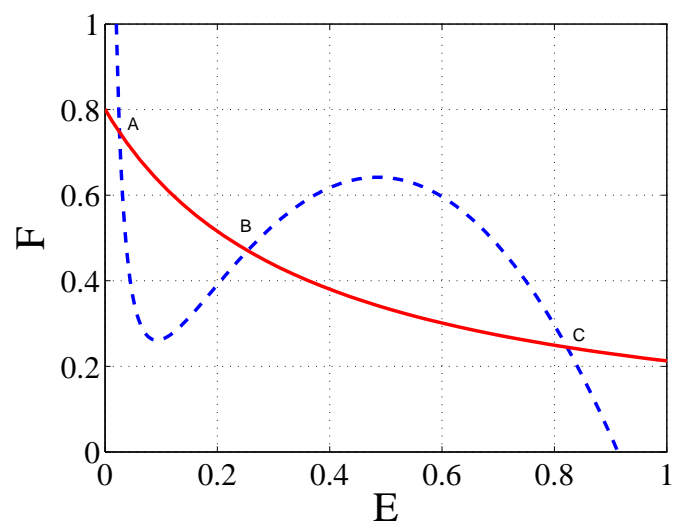

FIG. 2.4. Phase portrait of system (2.5). Dashed and solid curves are the nullclines associated with the first and the second equations of system (2.5), respectively. Points $A, B$ and $C$ are stationary points of system (2.5), points $A$ and $C$ are stable nodes, point $B$ is a saddle.

Let us stress out the conditions that determine erythroid progenitor fate. If during the cell cycle, the value of Erk becomes greater than a given critical value $E_{c}$, the cell self-renews. At the end of the cell cycle, it divides and gives rise to two daughter cells identical to the mother cell. However, if Fas concentration becomes greater than a given critical value, $F_{c}$, which depends on Epo concentration, the cell immediately dies by apoptosis. If neither of these conditions is satisfied, the cell differentiates. At the end of the cell cycle, the cell divides and gives birth to two more mature cells labeled here as reticulocytes.

Once produced, reticulocyte remain in the computational domain during a time equal to one cell cycle, during which it produces Fas-ligand. Then it becomes a mature erythrocyte and enters the bloodstream. In the framework of our model, reticulocytes that have performed one cell cycle are removed from the computational domain.

The intracellular regulation scheme described above concerns erythroid progenitors but not reticulocytes. Indeed, reticulocytes are post-mitotic cells and do not proliferate. However, reticulocytes produce Fas-ligand, which influences intracellular regulation of erythroid progenitors (the second equation in system (2.5) depends on Fas-ligand). For large values of Fas-ligand, trajectories of the system move towards greater values of $F_{i}$ and to smaller values of $E_{i}$.

Extracellular regulation. Reticulocytes (post-mitotic precusor cells) produce the protein Fas-ligand, which binds to Fas receptors located on the surface of neighbouring erythroid progenitors, and stimulates apoptosis or differentiation. Fas-ligand distribution in space is modelled the following reaction-diffusion equation,

$$
\frac{\partial F a s L}{\partial t}=D \Delta F a s L+W-\sigma F a s L
$$

where $W$ is a source term proportional to the local concentration of reticulocytes. We have chosen a small diffusion coefficient to account for the short-range cell-cell interaction [12]. Short diffusion means that Fas-ligand influences erythroid progenitors only when they are sufficiently close to reticulocytes, mimicking cell-cell contact interaction. Parameter values are in Table 2.1.

The source term $W$ in equation (2.6) depends on the spatial distribution of reticulocyte. In numerical simulations, the local concentration of reticulocytes is computed 


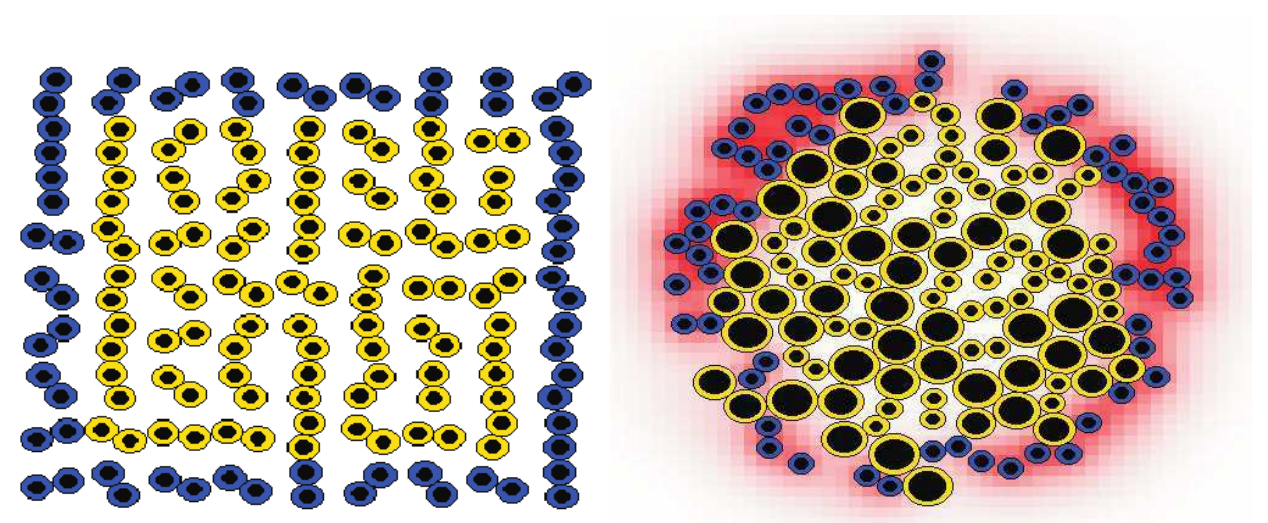

FIG. 2.5. Erythroblastic island: yellow cells in the center are erythroid progenitors, blue cells at the border are reticulocytes producing Fas-ligand (in red). The initial cell distribution is on the left panel, the typical configuration of the island after several cell cycles on the right panel.

as the total cell area in each box of the numerical grid (see Section 2.1). The spaceand time-dependent concentration of Fas-ligand, FasL $(x, t)$, enters the second equation of the system (2.5) with the value $\operatorname{Fas} L\left(x_{i}, t\right)$, where $x_{i}$ is the center of cell $i$. That is, cell $i$ "sees" the FasL local concentration at its center.

Figure 2.5 shows the evolution of an erythroblastic island under regulation of Fas-ligand (Epo concentration is constant). The left panel represents the initial cell configuration with immature progenitors inside and more mature reticulocytes outside. The right panel shows the typical structure of the island several cell cycles later. Extracellular Fas-ligand concentration (shown in red color) is higher near reticulocytes. Fas-ligand released by reticulocytes bind to Fas receptors located on the membrane of nearby erythroid progenitors. This, in turn, stimulates the production of Fas and, as a consequence, the intracellular concentration of Fas in the progenitor can be sufficiently high to induce its apoptosis. Progenitors located at some distance from reticulocytes can have an intermediate value of intracellular Fas, high enough to prevent Erk from being activated but low enough for the progenitor to survive. Such cells will preferentially differentiate into reticulocytes. Finally, progenitors located far from reticulocytes mostly self-renew, due the the antognistic activation of Erk.

A direct consequence of this description is that the evolution of an erythroblastic island depends on its size and the number of reticulocytes. In a small island, less progenitors will self-renew and the island will disappear after a couple of cell cycles. In contrast, if the island is sufficiently large, more progenitors will self-renew and the island will grow. Typical examples (obtained through 40 simulations, with the quartiles represented by dashed lines) are shown in Figure 2.6 where upper curves represent the number of progenitors, lower curves the number of reticulocytes. Figure 2.6a shows normal erythropoiesis with approximately stable number of progenitors. Although in some simulations cell population vanishes or grows infinitely, the average number of progenitors across 40 simulations is approximately constant (Figure 2.6a).

2.3. Pharmacokinetics and pharmacodynamics of Ara-C. Cytosine arabinoside, or Ara-C, is one of the most common and effective chemotherapeutic agents against acute myelogenous leukemia. Once introduced intravenously in the organism, Ara-C penetrates cell membranes by a mechanism based essentially on the human 


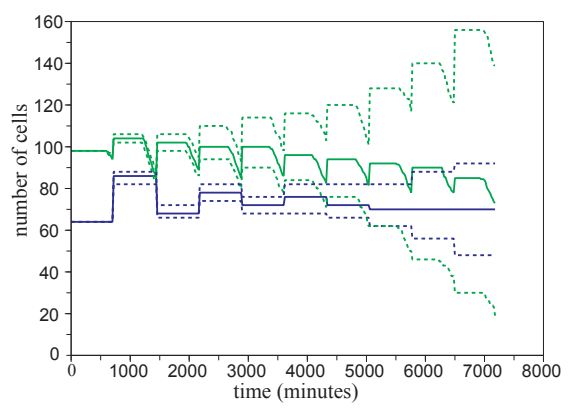

(a) Normal erythropoiesis

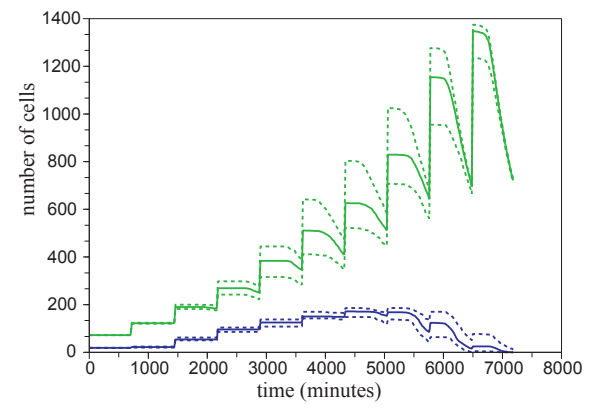

(b) Excessive self-renewal

FIG. 2.6. Time evolution of cell number in an erythroblastic island. Solid curves indicate means and dotted lines the quartiles over 40 simulations. Upper curves represent the number of progenitors. Lower curves represent the number of reticulocytes.

equivibrative nucleoside transporter-1 (hENT1), which can be considered as facilitated diffusion. This process does not need energy and follows the gradient of concentration up to the equilibrium between the intracellular and extracellular concentrations of Ara-C.

membrane

$\sim$ DNA

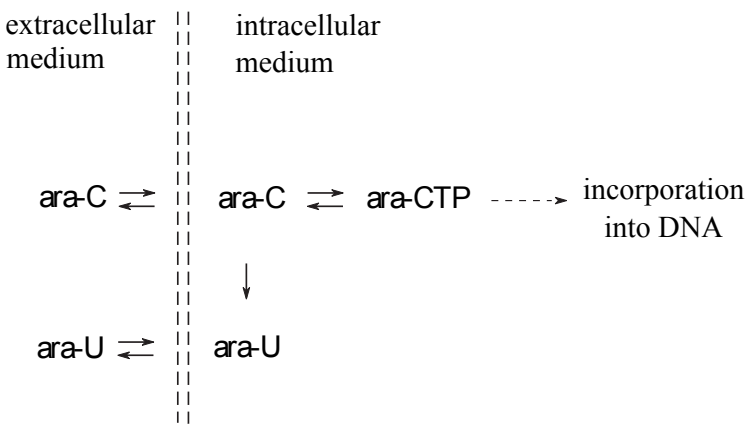

Fig. 2.7. Metabolism of Ara-C. Schematical representation of Ara-C pharmacokinetics: Ara-C crosses cell membrane, it is phosphorylated becoming Ara-CTP. Ara-CTP is used for DNA replication instead of nucleotide C. Besides, Ara-C can be transformed in Ara-U. Adapted from Morrison et al. [33].

Inside cells, part of Ara-C is phosphorylated into its active form, Ara-CTP, which arrests DNA synthesis and induces cell apoptosis. The rest, about $70 \%$ of Ara-C, is deaminated into its inactive form Ara-U. Ara-CTP, in turn, can be dephosphorylated back to Ara-C. In order to take into account the intracellular kinetics of Ara-C and Ara-CTP wa adapted a model from Morrison and colleagues. This model and parameter values (given in Table 2.1) were validated against experimental data [33]. We take into account these three reactions in order to model the intracellular kinetics of Ara-C and Ara-CTP (Figure 2.7). The phosphorylation rate of Ara-C into Ara-CTP, 
denoted by $r_{p}$, is given by a Michaelis-Menten equation

$$
r_{p}=\frac{V_{k}}{1+\frac{K_{m} K_{a}}{w_{i}}},
$$

where $w_{i}$ is the Ara-C concentration inside the cell $i, K_{m}$ is the Michaelis-Menten constant, $K_{a}$ accounts for additional effects and $V_{k}$ is the kinase activity responsible for phosphorylation.

Denote by $w_{i}^{a}$ the Ara-CTP concentration in the cell $i$. Ara-CTP dephosphorylation rate, $r_{d p}$, is given by

$$
r_{d p}=\frac{V_{d p}}{1+\frac{\alpha^{1} K_{d p}}{w_{i}^{a}}},
$$

with $V_{d p}$ the enzyme activity responsible for dephosphorylation, $\alpha^{1}$ accounts for additional effects, $K_{d p}$ is the Michaelis-Menten constant. The deamination rate $r_{d a}$ of Ara-C follows a similar kinetics,

$$
r_{d a}=\frac{V_{d a}}{1+\frac{K_{d a}}{w_{i}}}
$$

where $V_{d a}$ is the enzyme activity and $K_{d a}$ the Michaelis-Menten constant.

The kinetics of intracellular Ara-C concentration in cell $i$ is then described by the following equation,

$$
\frac{d w_{i}}{d t}=k_{1}\left(w-w_{i}(t)\right)-k_{2} w_{i}(t)-\left(r_{p}-r_{d p}+r_{d a}\right),
$$

where $w=w(t)$ is the time-dependent extracellular concentration of Ara-C, assumed to be uniformly distributed in the bone marrow. All cells are in contact with AraC. The constant $k_{1}$ is related to the rate of membrane penetration, $k_{2}$ accounts for degradation of Ara-C. There is no extracellular degradation of Ara-C. We reproduce a chronomodulated treatment protocol where cells are infused in Ara-C for two hours, then Ara-C is removed (or washed out). This is modelled by setting the extracellular Ara-C profile $w(t)$ to a constant $w_{T}>0$ for two hours after the starting time of administration, and to zero outside the treatment time. This Ara-C profile is repeated at fixed intervals $T$, ranging from 8 to 74 hours. Individual dosages $w_{T}$ are adjusted as a function of $T$ to keep the total amount of Ara-C delivered constant, i.e. $w_{T} / T$ is constant. The time of the day of the first treatment is $t_{0}$. Thus $w(t)$, with time $t$ in hours, is given by

$$
w(t)= \begin{cases}w_{T} & \text { if } t_{0} \leq t \bmod T \leq t_{0}+2 \\ 0 & \text { otherwise }\end{cases}
$$

The balance equation for the intracellular concentration of Ara-CTP is

$$
\frac{d w_{i}^{a}}{d t}=k_{\alpha}\left(r_{p}-r_{d p}\right)
$$

Ara-CTP exerts a cytotoxic effect on all dividing cells when it gets inserted into replicating DNA, replacing natural nucleotides. It arrests DNA synthesis and induces apoptosis. The quantity $p_{i}$ represents the amount of Ara-CTP incorporated into DNA. 


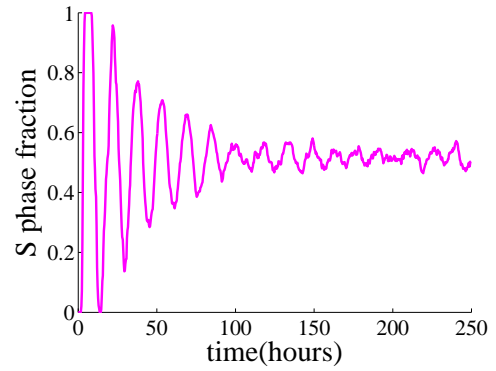

(a) $G_{1 \max }=7$ hours

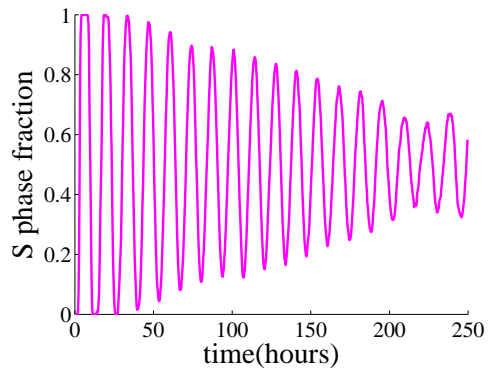

(b) $G_{1 \max }=3$ hours

FIG. 2.8. Proportion of cells in phase $S$, with $G_{1 \min }=0$.

The rate of Ara-CTP incorporation into replicating DNA follow a linear equation during the $\mathrm{S}$ phase, where it is assumed that DNA is replicated at a constant rate,

$$
\frac{d p_{i}}{d t}=k_{\beta} w_{i}^{a},
$$

with $k_{\beta}$ the rate of insertion of Ara-CTP. We assume that apoptosis is triggered when the amount of Ara-CTP into DNA reaches a critical value $p_{c r}$.

2.4. Organization and duration of the cell cycle. The cell cycle proceeds sequentially through four phases, the $G_{1}, S, G_{2}$ and $M$ phases. The DNA synthesis $S$ phase and the mitosis $M$ phase are separated by the $G_{2}$ phase (for gap) during which the cell checks for DNA damage and decides whether it progresses to mitosis. After mitosis, proliferating cells enter the $G_{1}$ phase, where it grows and wait for signal to progress through $\mathrm{S}$ phase. After mitosis, cells can also enter a quiescent state, denoted $G_{0}$, during which cells are non-cycling. The duration of the $G_{0}, G_{1}$ and $G_{2}$ phases are controlled by various intracellular and extracellular mechanisms. Erythroid progenitors are assumed to be continuously cycling, with a non-existent $G_{0}$ phase, while reticulocytes are in $G_{0}$ phase. The $G_{1}$ phase accounts for most of the cell cycle length variability [36]. We assume that the $G_{1}$ phase duration is a random variable with a uniform distribution between $G_{1 \min }$ and $G_{1 \max }$, the minimal and maximal duration.

From a modelling point of view, variation in the $G_{1}$ phase duration, and consequently in cell cycle duration, cause cells to desynchronize, i.e. the phase distribution of cells is constant in time. This is an essential feature for the correct modelling of Ara-C pharmacodynamics and treatment of leukemia. We ran two simulation of the complete model, with $G_{1 \min }=0$ and $G_{1 \max }=3$ or 7 hours, with initially synchronized cells. For larger cell cycle variability $\left(G_{1 \max }=7\right.$ hours $)$, the cell population desynchronizes and the proportion of cells in $S$ phase becomes almost constant after approximately 7 cell cycles (Figure $2.8 \mathrm{a})$. For lower cell cycle variability $\left(G_{1 \max }=3\right.$ hours), the proportion of cells in $S$ phase is strongly oscillating, even after several cell cycles, and the population is not desynchronized.

2.5. Circadian rhythm in the cell cycle. Circadian rhythms gate the progression through the cell cycle, and hence modulates the proportion of cells in the $S$ phase according to the time of day [39]. Therefore, the efficiency of a cell cycle-specific drug can also depend on time of administration [2]. to describe circadian rhythms, 
TABLE 2.1

Parameter values (N.U. means "no unit", min is for "minute"). All simulations use these parameter values, unless explicitly indicated.

\begin{tabular}{|c|c|c|c|c|}
\hline Parameter & & Value & Unit & Ref/Comment \\
\hline & "Mechanical forces & & & \\
\hline$r_{0}$ & Cell radius & 4 & $\mu \mathrm{m}$ & \\
\hline$H_{1}$ & Cell compressible part & 2 & $\mu \mathrm{m}$ & \\
\hline$K$ & Positive parameter & 1000 & $\mu \mathrm{m} \cdot \min ^{-2}$ & \\
\hline \multirow[t]{2}{*}{$\mu$} & viscosity & 10000 & $\min ^{-1}$ & \\
\hline & Intracellular regulation & & & {$[16]$} \\
\hline$\alpha$ & Epo-dependent Erk activation rate & 0.0003472 & $\min ^{-1}$ & \\
\hline$k$ & Erk self-activation power & 2 & N.U. & \\
\hline$\beta$ & Erk self-activation rate & 0.05 & $\min ^{-1}$ & \\
\hline$a$ & Erk degradation rate & 0.004 & $\min ^{-1}$ & \\
\hline$b$ & Erk inhibition constant & 0.0138 & $\min ^{-1}$ & \\
\hline$\gamma_{0}$ & Constant Fas activation rate & 0 & $\min ^{-1}$ & \\
\hline$k_{\gamma}$ & Fas-ligand-dependent Fas activation rate & 0.0002 & $\min ^{-1}$ & \\
\hline$c$ & Fas inhibition rate & 0.00138 & $\min ^{-1}$ & \\
\hline$d$ & Fas degradation rate & 0.0001 & $\min ^{-1}$ & \\
\hline$E_{c}$ & Erk critical level & 0.4 & N.U. & \\
\hline \multirow[t]{2}{*}{$F_{c}$} & Fas critical level & 0.2 & N.U. & \\
\hline & Extracellular regulation & & & \\
\hline$D$ & Fas-ligand diffusion coefficient & $1 e^{-5}$ & $\mu \mathrm{m}^{2} \cdot \min ^{-1}$ & {$[12]$} \\
\hline$\sigma$ & Fas-ligand degradation rate & 0.02 & $\min ^{-1}$ & \\
\hline \multirow[t]{2}{*}{$W$} & Source term & 0.0001 & $\min ^{-1}$ & \\
\hline & Metabolism of AraC & & & {$[33]$} \\
\hline$k_{1}$ & Membrane penetration rate & 0.0116 & $\min ^{-1}$ & \\
\hline$k_{2}$ & Ara-C degradation rate & 0 & $\min ^{-1}$ & \\
\hline$V_{k}$ & Kinase activity & 1.28 & $\mu \mathrm{M} . \min ^{-1}$ & \\
\hline$K_{m}$ & Michaelis-Menten constant & 27.0 & $\mu \mathrm{M}$ & \\
\hline$K_{a}$ & Additional effects constant & 16 & N.U. & \\
\hline$V_{d p}$ & Enzyme activity & 300 & $\mu \mathrm{M} \cdot \min ^{-1}$ & \\
\hline$K_{d p}$ & Michaelis-Menten constant & 900 & $\mu \mathrm{M}$ & \\
\hline$\alpha^{1}$ & Additional effects constant & 44.4 & N.U. & \\
\hline$V_{d a}$ & Enzyme activity & 16.45 & $\mu \mathrm{M} \cdot \min ^{-1}$ & \\
\hline$K_{d a}$ & Michaelis-Menten constant & 1011.7 & $\mu \mathrm{M}$ & \\
\hline$k_{\alpha}$ & Metabolism constant & 0.8 & N.U. & \\
\hline$k_{\beta}$ & Ara-CTP conversion rate & 1 & N.U. & \\
\hline$p_{c r}$ & Critical value of Ara-CTP in DNA & 40 & $\mu \mathrm{M}$ & \\
\hline \multirow[t]{3}{*}{$w_{T}$} & extracellular Ara-C concentration & 10 & $\mu \mathrm{M}$ & for $T=8$ hours \\
\hline & & & & $w_{T}=1.25 \mu \mathrm{M} / \mathrm{h} \times T$ \\
\hline & Circadian rhythm & & & \\
\hline$c_{x}$ & Constant characterizing the circadian clock & 0 & N.U. & $\begin{array}{l}c_{x}=0.5 \text { if } \\
\text { circadian regulation }\end{array}$ \\
\hline \multirow[t]{2}{*}{$\theta$} & Phase shift & 5 & hours & \\
\hline & Cell cycle & & & \\
\hline$G_{1 \min }$ & Minimal duration of phase $G_{1}$ & 5 & hours & \\
\hline$G_{1 \max }$ & Maximal duration of phase $G_{1}$ & 31 & hours & \\
\hline$S$ & Duration of phase $S$ & 10 & hours & \\
\hline \multirow[t]{2}{*}{$G_{2} / M$} & Duration of phase $G_{2} / M$ & 3 & hours & \\
\hline & "Cell cycle (leukemic haematopoiesis) & & & \\
\hline$G_{1 \max }$ & Leukemic stem cells & 31 & hours & \\
\hline$G_{1 \max }$ & proliferative leukemic cells & 12 & hours & \\
\hline
\end{tabular}


we introduce a "timer" variable $c y c D$, which is an abstraction of the evolution of the expression of cyclin D1, a circadian clock-controlled protein, whose activity is required for cell cycle $G_{1} / S$ transition. When the timer $c y c D$ reaches the threshold value $c y c D_{c}$, the cell enters $S$ phase. The $G_{1}$ phase consists of a incompressible length $G_{1 \text { min }}$ and a variable duration. We label the beginning of the variable length part of $G_{1}$ phase $t_{v}$ (for a cell having divided a time $G_{1 \text { min }}$ earlier), and we prescribe $c y c D\left(t_{v}\right)=c y c D_{v}$, where the initial concentration of the protein $c y c D_{v}$ is a random variable uniformly distributed in $\left[0, c y c D_{c}\right]$.

Since the cyclin D1 corresponding gene is controlled by the circadian rhythm, cyclin D1 protein expression is determined by the time of the day. This is reflected in the following equation for the concentration of the protein,

$$
\frac{d c y c D}{d t}=1+c_{x} \sin (f(t-\theta))
$$

where $f=\frac{2 \pi}{24}, c_{x} \in[0,1]$ is the amplitude to mean ratio of the circadian rhythms and $\theta$ is a phase shift chosen to reproduce circadian timing of cell division in humans. The solution to (2.11) is given by the formula:

$$
\operatorname{cyc} D(t)=c y c D_{0}+\left(t-t_{0}\right)+\frac{c_{x}}{f} \cos \left(f\left(t_{0}-\theta\right)\right)-\frac{c_{x}}{f} \cos (f(t-\theta)),
$$

We set $\tau=t-t_{0}$, where $\tau$ is the time spent in the variable length part of $G_{1}$ phase. Then we have

$$
c y c D\left(t_{0}+\tau\right)=c y c D_{0}+\tau+\frac{c_{x}}{f} \cos \left(f\left(t_{0}-\theta\right)\right)-\frac{c_{x}}{f} \cos \left(f\left(t_{0}+\tau-\theta\right)\right),
$$

If, $c y c D\left(t_{0}+\tau_{c}\right)=c y c D_{c}$, the concentration of the protein reaches the $G_{1} / S$ transition threshold value, and the cell enters the $S$ phase. That way, $G_{1}$ phase duration is $G_{1 \min }+\tau_{c}$. The variable duration $\tau_{c}$ a random variable distributed in $\left[0, \tau_{\max }\right]$ where $\tau_{\max }=\tau_{\max }\left(t_{0}-\theta\right)$ is the solution in $\tau$ to equation (2.13) with $c y c D_{0}=0$. The maximal value of $\tau_{c}, \tau_{\max }$, depends on the difference between the phase of the circadian clock and the time at which the cell last divided. In absence of circadian rhythms $\left(c_{x}=0\right)$, we obtain $\tau_{\max }=c y c D_{c}$. To remain consistent with the $G_{1}$ phase duration in absence of the clock, we set $c y c D_{c}=G_{1 \max }-G_{1 \min }$.

2.6. Ara-C treatment applied to leukemic haematopoiesis. When we study Ara-C treatment of the AML, we first consider the case where leukemic stem cells self-renew but they cannot differentiate or die by apoptosis (Section 3.2). We call this model the simplest model of leukemia. In reality, leukemic hematopoiesis can be more complex. It starts with leukemic stem cells, which possess the property of self-renewal, followed by partially differentiated leukemic cells, some of them proliferating while some others do not proliferate. The exact organization of leukemic hematopoiesis may strongly depend on individual patients, and it is very difficult, if possible, to determine it clinically. Therefore comparison of mathematical modeling with available clinical data can give some insight on leukemic hematopoiesis. We will discuss this question in more detail in Section 3.3. We will suggest a model of leukemic hematopoiesis in order to describe the clinical data presented in [31].

Let us note that various models of tumor stem cells have been proposed, both in solid tumors and in hematological malignancies such as acute myeloid leukemia. In our approach we have considered that all leukemic cells, including those that undergo partial differentiation, originate from a single precursor. 
3. Results. In this section, we run simulations of the hybrid model presented in Section 2. We simulate the action of Ara-C on two type of erythroblastic islands, with either normal or leukemic erythroid progenitors. In Section 3.1, we focus on the effect of treatment on proliferation and survival of normal cells. We investigate the respective influences of the period of treatment and the starting hour of treatment with respect to the cell cycle duration. In Section 3.2, we simulate the effect of a chronomodulated treatment with Ara-C, in the situation where normal cells are subject to circadian rhythm $\left(c_{x}=0.5\right.$ in (2.13)) whereas leukemic cells do not exhibit circadian rhythm dependency $\left(c_{x}=0\right)$. In these two cases, the cell population is first simulated from an initial state until it reaches a satisfactory state, in which the erythroblastic island is large enough and cells are well mixed, as in the right panel of Figure 2.5. Consequently, time $t=0$ corresponds to the time of the beginning of the numerical experiments and not as the beginning of erythroblastic island simulations. In Section 3.3 we simulate leukemic haematopoiesis and its treatment with Ara-C.

3.1. Chronomodulated Ara-C treatment in a non-rhythmic population. We first consider a population of erythroid cells, composed of two types of cells: erythroid progenitors and reticulocytes, and apply an Ara-C treatment protocol as described in Section 2.3. We investigate the relevance of taking cell cycle durations into account when treating with Ara-C, when the circadian clock is neglected $\left(c_{x}=0\right)$.

We performed numerical simulations of erythroid cell populations with an average duration of cell cycle equal to 31 hours under Ara-C treatment. We considered three different administration intervals $T=26,34$ and 64 hours (Figure 3.1a). For each treatment interval, we corrected the dosage $w_{T}$ so as to keep to total amount of Ara$\mathrm{C}$ administered constant (constant area under the curve of $w(t)$ ). Depending on the treatment intervals, the cell population can either disappear (Figure 3.1a) or grow (Figure 3.1b,c). The interval $T=26$ hours was most lethal to the cells, while the interval $t=36$ hours was least lethal to the cells. Therefore the growth rate under periodic Ara-C treatment is not a monotonous with respect to the treatment interval.

Because the cytotoxic effect of Ara-C is related to DNA synthesis, it is expected that a periodic treatment will partly synchronize the cell population. As a result, time-periodic drug administration killing cells in the $S$ phase will affect the phase distribution of subsequent cell generations. This can result in so-called resonance effect, as discussed in [13, 21]. Resonance theory predicts that when the treatment period is close to the duration of the cell cycle or its multiples, cell kill is minimised. This is because cells that are not in the $S$ phase during one administration will be less likely to be in $S$ phase at subsequent administrations, and are shielded from the treatment.

To further explore this idea, and to see whether a resonance effect is seen in the hybrid model, we computed the growth rate of the erythroblastic island under periodic treatments with periods ranging from $T=20$ to 74 hours. The resonance theory predicts that the growth rate, as a function of $T$, should show a local maximum near multiples of the cell cycle length. To test that, we simulated periodic treatment in two populations with different cell cycle length, the first one with a cell cycle length of $31.0 \pm 13.0$ hours and the second one, $33.5 \pm 15.5$ hours. Cell population growth or decay was approximately exponential, with periodic modulations due to the action of the treatment. We assumed that cell number evolved as $N(t) \sim C(t) e^{\lambda t}$, where $\lambda$ is the growth exponent (either positive or negative) and $C(t)$ is a $T$-periodic function, and fitted $\lambda$ (Figure 3.2). As a function of $T, \lambda$ showed a peak near the cell cycle length. In the first population (length 31.0 hours), the resonance effect was strong, 


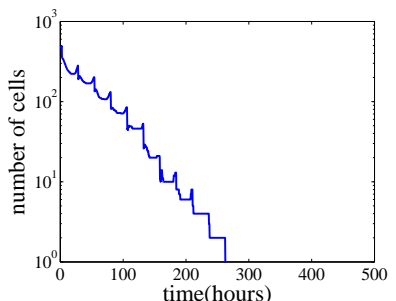

(a) Injection of $A r a C$ every 26 hours.

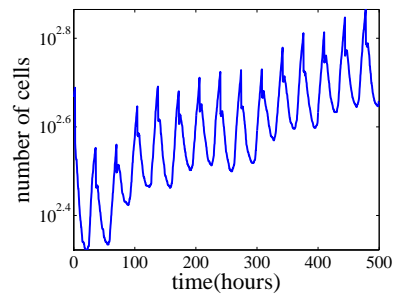

(b) Injection of $\mathrm{AraC}$ every 34 hours.

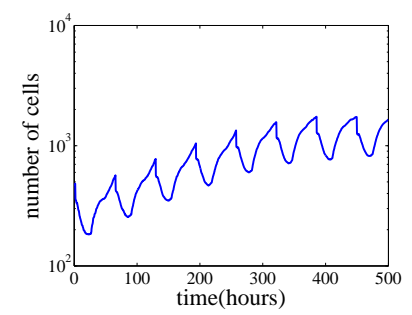

(c) Injection of AraC every 64 hours.

FIG. 3.1. Time evolution of erythroid cell number under treatment with Ara-C, for three different treatment intervals $T=26$ hours (a), $T=34$ hours (b), and $T=64$ hours (c).

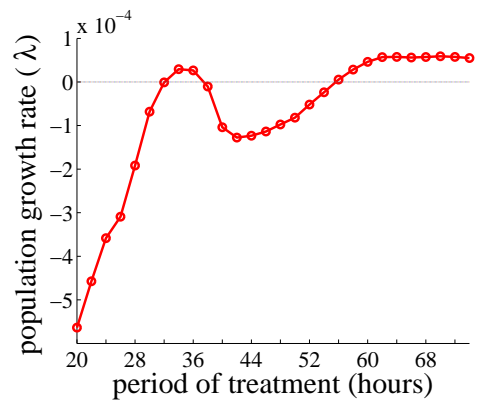

(a) $G_{1 \max }=31 \mathrm{hr}$

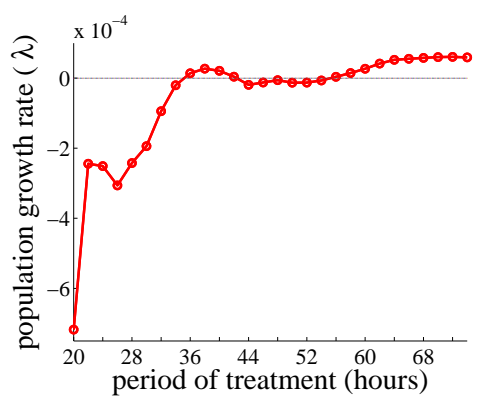

(b) $G_{1 \text { max }}=36 \mathrm{hr}$

FIG. 3.2. Growth exponent $\lambda$ as a function of the treatment period $T$, for two different cell cycle lengths, $31.0 \pm 13.0$ hours (a), and $33.5 \pm 15.5$ hours (b).

and $\lambda$ peaked at around 33.0 hours (Figure 3.2a). In the second one (length 33.5), $\lambda$ peaked at around 38.0 hours, but the resonance effect was much less pronounced (Figure 3.2b). For both populations, peaks at higher multiples of the cell cycle lengths were almost absent. This is probably due to the fast desynchronization of the cell cycle in these two populations, whose lengths range between 18.0 and 44.0 hours, and 18.0 and 49 hours respectively. Greater cell cycle variability might also account for the weaker resonance effect in the second population. Taken together, these results indicate that resonance is seen in the hybrid model, but that cell cycle variability reduces it effect on the growth rate.

3.2. Chronomodulated Ara-C treatment in a circadian population. Our simulation results with the hybrid model confirmed that the search for an optimal treatment schedule is relevant within the context of erythroid cell populations. The progenitor population can either grow or decay, depending on the Ara-C treatment period. Here, we look at the effect the circadian modulation of cell cycle progression has on the treatment efficacy.

Most cytotoxic drugs used in chemotherapy affect both normal and leukemic cells. In leukemia, downregulation of circadian clock genes is often observed [40], and malignant cells can even escape circadian control [41]. Thus, a treatment protocol based on chronotherapeutic principles could exploit this difference between normal and leukemic cells to reduce toxicity (chronotolerance) and to increase efficacy (chro- 


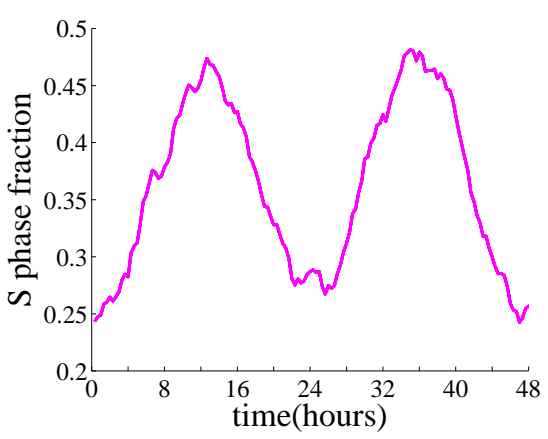

FIG. 3.3. Time evolution of the fraction of proliferating cells in $S$ phase, under circadian clock regulation $\left(c_{x}=0.5\right)$.

noefficacy).

We consider two different populations. The first one consists of erythroid progenitors under circadian control $\left(c_{x}=0.5\right)$. Simulations in absence of treatment shows that circadian control naturally induces temporal synchronization of DNA synthesis activity (Figure 3.3). The fraction of erythroid progenitors in the $S$ phase is maximal around 13:00 and minimal between 0:00 and 01:00, in agreement with clinical observations [39]. The second population consists of leukemic cells, without circadian control $\left(c_{x}=0\right)$, and therefore desynchronized.

We first submitted the normal population (circadian clock-regulated) to chronomodulated Ara-C treatments with intervals $T$ ranging from 8.0 to 70.0 hours, and with first treatment starting at times $t_{0}$ between 01:00 and 24:00, and looked at the outcome in terms of growth exponents $\lambda$ (Figure 3.4). High values of $\lambda$ were associated to a good outcome (low toxicity) while low values of $\lambda$ were associated to bad outcomes (high toxicity). The most important variations of the outcome as a function of $t_{0}$, for fixed $T$ were observed for treatment intervals $T=24$ hours (Figure 3.4b, zoom in of Figure 3.4a) and $T=48$ hours (Figure 3.4c, zoom in of Figure 3.4a). Outside these intervals, the starting delivery time $t_{0}$ does not appear to modify the outcome of the simulations, which depend mostly on $T$ (Figure 3.4a). If the interval $T$ is slightly larger (less, resp.) than 24 hours or 48 hours, the time of first delivery $t_{0}$ should be advanced (delayed, resp.) to produce an equivalent outcome. This dependence is more best expressed at 48 hours (Figure 3.4c), and is consistent with previous results [4]. The white area between 30 and 40 hours (Figure 3.4a) corresponds to a treatment interval close to the average duration of the cell cycle. The reason of its appearance is the same as for the first peak in Figure 3.2a, discussed in the Section 3.1. This shows that the cell cycle resonance effect can be present even when the cycle cell is driven by the circadian clock. Taken together, these results show that the circadian clock influence the treatment outcome when the intervals are close to multiples of the circadian period. Outside these intervals, the timing $\left(t_{0}\right)$ of the treatment has no effect on the outcome.

We then compared the evolution of cell numbers in the normal population, subject to the same chronomodulated Ara-C treatment with intervals $T=24$ or 48 hours, treatment times $t_{0}$ equal to 01:00 or 13:00. The time 01:00 corresponds to the lowest fraction of cells in the $S$ phase, while 13:00 corresponds to the highest fraction (see Figure 3.3). Cell number decay was slower when Ara-C was administered at 01:00 compared to 13:00 (Figure 3.5), whether the treatment was administered with 24 


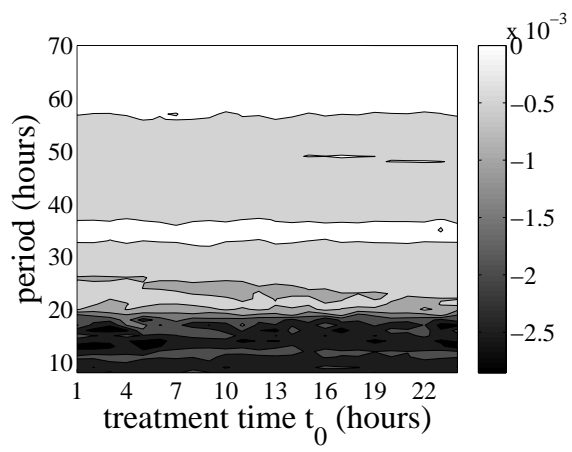

(a)

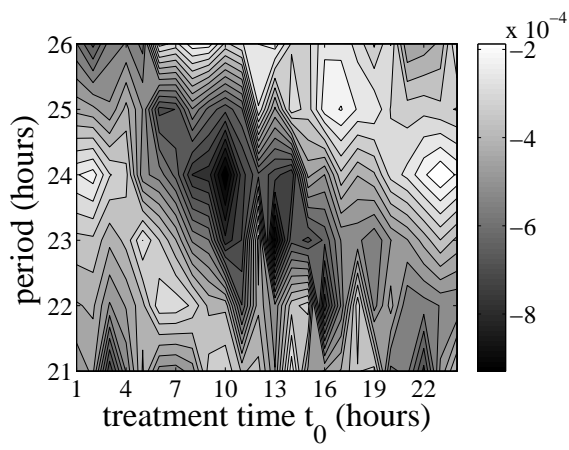

(b)

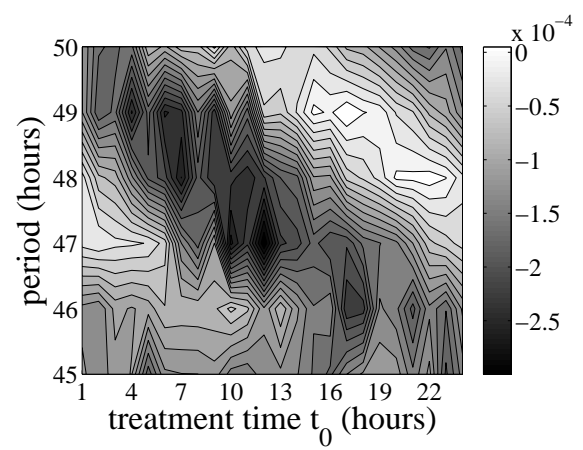

(c)

FIG. 3.4. Treatment outcomes for normal cells $\left(c_{x}=0.5\right)$. The gray scale shows the value of $\lambda$ as a function of the first day delivery time $t_{0}$ and the interval $T$ between two consecutive drug administrations. (a) Growth exponent $\lambda$ for $t_{0}$ ranging from 1 a.m. to midnight, and $T$ ranging between 8 and 65 hours. (b) Specific region around period T=24 hours. (c) Specific region around period $T=48$ hours, respectively. All three panels correspond to the same numerical simulations. Low values of $\lambda$ indicate bad treatment outcomes (high toxicity, dark regions) and high values indicate good treatment outcome (low toxicity, light regions).

(Figure 3.5a) or 48 hour-intervals (Figure 3.5b). Decay was slower with treatments at $T=48$ hours than $T=24$ hours, independently from $t_{0}$. Therefore, the best outcome (less toxicity) is at $T=48$ hours with a $t_{0}$ of 01:00. The worst outcome is at $T=24$ hours with a $t_{0}$ of 13:00.

We finally compared the outcome of normal cells and leukemic cells. We first assume that the leukemic population has the same properties than the normal one, except that it is not influence by the circadian clock. All other properties and parameter values are identical. Populations of normal and leukemic cells were subjected to a 48 hour-interval treatment at 01:00 or 13:00 (Figure 3.6). The population of normal cells slowly decays while the population of leukemic cells dies out when $t_{0}$ is 01:00 (Figure 3.6a). In contrast, the population of normal cells dies much faster than the population of leukemic cells when $t_{0}$ is 13:00 (Figure 3.6b). These results indicate that when treating at multiples of the circadian periods, the exact timing of administration can decrease Ara-C toxicity in normal cells, while maintaining the same efficacy against leukemic cells with a fixed set of parameter values (dashed curves in Figure 3.6a $\left(G_{1 \max }=31 \mathrm{~h}\right)$ and in Figure 3.6b). However, leukemic cell kinetics varies between cells and between patients. Varying the value of $G_{1 \max }$, we looked at 


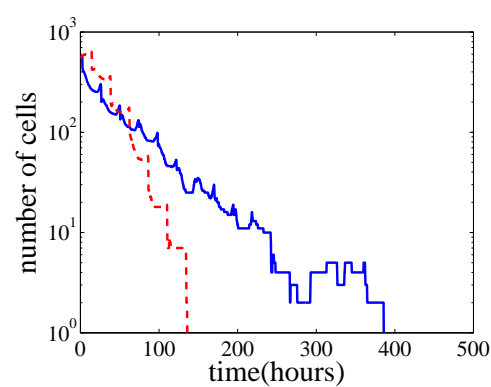

(a)

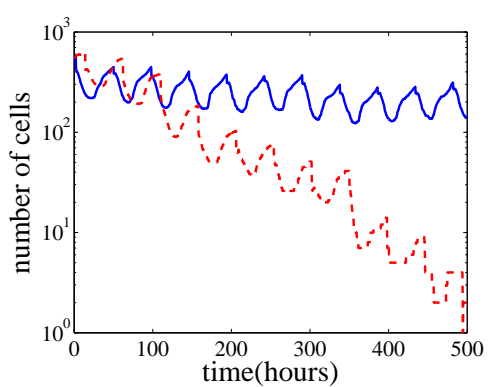

(b)

FIG. 3.5. Time evolution of the total number of normal cells $\left(c_{x}=0.5\right)$ with Ara-C administration at 01:00 (solid lines) or at 13:00 (dashed lines) with a treatment interval $T=24$ hours (a), or $T=48$ hours (b).

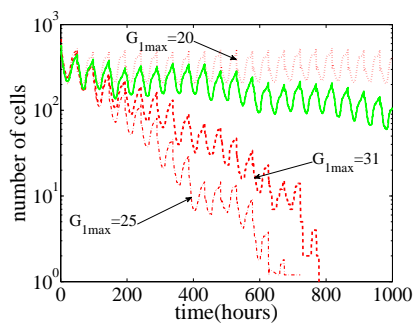

(a)

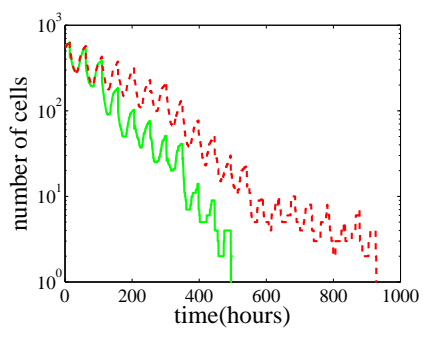

(b)

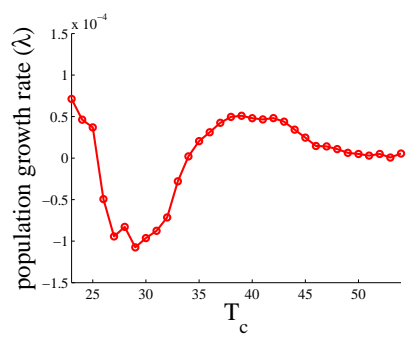

(c)

Fig. 3.6. Time evolution of the total number of normal cells (solid green curve, $c_{x}=0.5$ ) and leukemic cells ( dashed red curve, $c_{x}=0$ ) with Ara-C administered every 48 hours at 01:00 (a), or at 13:00 (b). Populations of leukemic cells with different cell cycle length, where $G_{1 \max }$ is varied, are presented (a). Growth exponent $\lambda$ as a function of cell cycle length $T_{c}$ of leukemic cells (c).

the effect of cell cycle length on the leukemic cell exponent growth for cell cycle length between $23 \mathrm{~h}$ and $54 \mathrm{~h}$, subject to chronomodulated Ara-C (Figure 3.6c). For cell cycle length over $46 \mathrm{~h}, \lambda$ varies little. For shorter cell cycles, we expected larger values of $\lambda$, but we found instead a minimum at $T_{c}=28 \mathrm{~h}$, due to resonance. Therefore, cell populations with $T_{c}=28 \mathrm{~h}\left(G_{1 \max }=25 \mathrm{~h}\right)$ decrease faster than cell populations with $T_{c}=31 \mathrm{~h}\left(G_{1 \max }=31 \mathrm{~h}\right)$, as exemplified in Figure 3.6a. This shows that the efficacy of the treatment depends in a nonintuitive way on leukemic cell cycle kinetics.

3.3. Comparison with clinical data of leukemic haematopoiesis treatment. In the previous section we considered the simplest model of leukemia where 
leukemic stem cells self-renew but they cannot differentiate or die by apoptosis. Moreover this model does not take into account events occurring between the erythroblastic islands and the circulation, and does not track the number of circulating cells. Here we adapt this model to take these events into account and follow the number of circulating cells. This allows us to compare model simulations and clinical data on Ara-C treatment of leukaemia [31].

The protocol described in [31] was applied to a patient with acute myeloblastic leukemia who was unresponsive to conventional chemotherapy. Ara-C was administered as two hours continuous i.v. infusions for 5 times over a total duration of 34 hr with 6 -hr interval between infusions. The number of leukemic cells in blood was measured every 24 hours.

It appears that these data cannot be described with a single type of leukemic cells, and more complex leukemic hematopoiesis should be considered. We have tested different schemes of leukemic hematopoiesis. The minimal model includes leukemic stem cells (LSC), proliferative leukemic cells and mature leukemic cells (MLC) in blood. Apoptotic rate of LSCs is assumed to be zero. With probability 0.5, a leukemic stem cell after its division gives birth to two cells with higher degree of maturity, or to one LSC and one differentiated cell. These more mature cells, named here proliferative leukemic cells, represent the second type of cells in the model. Proliferative leukemic cells at different stages of maturation are also located in the bone marrow. Similar to LSCs, these cells do not die without medical treatment. After their division, they give two daughter cells with higher maturity. We assume here seven stages of their maturity. The cells of eighth stage of maturation form the third type of cells, so called post mitotic almost mature leukemic cells (MLC), which are released from the bone marrow into the blood. Parameter values of cell cycle duration for LSCs and proliferative leukemic cells are given in Table 2.1. The survival half-life of post mitotic mature leukemic cells in blood was estimated to be about $12 \mathrm{hr}$ [31]. It was implemented into the model by forcing post mitotic MLC to take a decision every 12 hours to die or to survive with probability 0.5 .

We take the pharmacokinetics of medical treatment into account and determine the number of leukemic cells in blood. In the case of leukemic hematopoiesis described above, simulations show a good agreement with the clinical data (Figure 3.7, solid curve). We note that leukemic cells persist in blood for about 60 hours after the treatment is finished. If we consider a model with only one type of leukemic cells (LSCs), then we cannot describe this effect because accumulation of leukemic stem cells in blood at the moment when the treatment is over will not be sufficient to provide this persistence (Figure 3.7, dashed curve). Including of post mitotic cells is crucial to achieve a good agreement with the clinical data.

4. Discussion. Erythropoiesis is the process of production and regulation of red blood cells, which occurs in discrete units of the bone marrow. To understand better this complex process, we proposed a new multiscale model of erythropoiesis in the erythroblastic island, based on hybrid discrete-continuous modelling. This approach takes into account several aspects, including: a) cells as individual objects that can divide, move, change their phenotype (differentiation), die, interact with each other and with the surrounding medium mechanically [22] and biochemically; b) intracellular regulatory networks responsible for cell fate, which also include the pharmacokinetics of medical treatment, described by ordinary differential equations; c) extracellular biochemical species, whose evolution are described by partial differential equations. Extracellular regulation includes local regulation due to cell communication in the tis- 


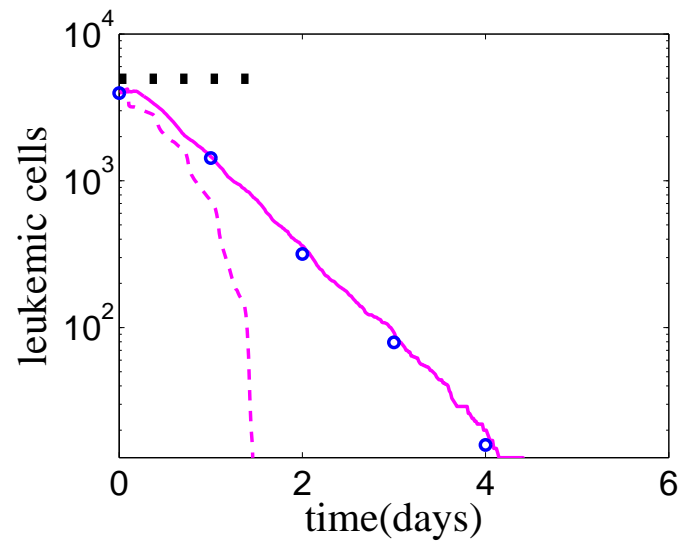

FIG. 3.7. The number of leukemic cells in blood during treatment. Magenta curves represent numerical simulations. Solid curve corresponds to leukemic haematopoiesis. Dashed curve corresponds to the hybrid model with one type of leukemic cells. Blue points represent the clinical data. Black intervals signify the time of treatment. $10 \mu \mathrm{m}$ of Ara-C was given as a 2-hr continuous i.v. infusion for 5 times over a total duration $34 \mathrm{hr}$ with 6-hr interval between the infusions.

sue and medical aspects such as treatment. Intracellular and extracellular regulations influence each other in a complex way to determine the fate of each single cells.

We applied this approach to study the treatment of acute myeloblastic leukemia (AML) with Ara-C, one of the main chemotherapeutic drugs used for AML patients. We observed a resonance effect in the survival of dividing cells undergoing chronomodulated treatment with phase-specific cytotoxic drugs. Such optimization of treatment was discussed before in [13, 21], where resonance effect was stronger as a result of low variability in the cell cycle duration. In vivo resonance effect is difficult to observe, perhaps due to heterogeneity in cell cycle times. Since Ara-C affects both normal and leukemic cells, one of the main questions in chemotherapy is to choose an optimal patient-adapted treatment protocol that allows elimination of most leukemic cells and preservation of maximum of normal cells. Our results indicate that chronotherapy, which takes into account circadian rhythms of normal cells, can improve the tolerance to the treatment.

There are numerous models devoted to modelling of leukemia and its treatment. However, most of them are population-based model, with little or no heterogeneity in tumor and host cells [14, 15]. Importance of heterogeneity is evidenced, for instance, by a recent phase III trial for chronotherapy [24], which showed a significant difference in outcome between women and men, something that was never modeled, or expected. One way to introduce this difference in a cell population is to take into account spatial cell distribution: cells differ by their position in space, that is by their environment (extracellular regulation), and also PK regulation and possibly circadian clock-regulated PK [3]. It influences intracellular regulation and cell fate. Recently, Altinok et al. [1,2] used a cellular automaton to explore the effect of the variability in the cell cycle length on chronotolerance and chronoefficacy of 5-FU and oxaliplatin. The model did not account for intracellular regulation which determine cell choice.

A recurring problem with large, detailed models is the question of parameter values. Here the hybrid model is modular, each module consisting of well defined models, with their own set of parameters. Pharmacokinetic, cell cycle, regulatory network kinetic parameters are known from the literature (Table 2.1). Some parameters are 
not directly available experimentally but we can still find them in order to reproduce biologically realistic functions of the system, such as correct proportions of each cell type. Among them are parameters for the intracellular regulation, which were chosen in order to describe a bistable switch inducing cell decision. Other parameter values can be identified. For example, $\theta$ was identified from the $S$ phase peak at 1 p.m.

The complex, multi-scale aspects of erythropoiesis modelling cannot be taken into account in simple models. In particular, we should consider spatial distributions of cells in order to take into account the influence of cell environment on its fate (the choice between self-renewal, differentiation and apoptosis). Moreover, the size of erythroblastic islands can determine their behavior. For instance, with the same values of parameters, a "small" island can decrease and disappear while a "large" one can grow. Simpler ODE models with average values of parameters cannot describe this effect. Continuous models based on partial differential equations can also be used to describe spatial inhomogeneities. However, continuous models are more difficult to justify formally than hybrid models. Usually, we first need to formulate a hybrid model. After that a continuous model can possibly be derived from it by some averaging or asymptotic procedure though this step can rarely be rigorously justified.

We note that extracellular regulation is not considered in the model of leukemic haematopoiesis. There are no extracellular signaling molecules (like Fas-ligand for erythroid progenitors), which influence cell fate. Since leukemic cells are not sensitive to normal regulatory mechanisms, we assume that they make their choice between selfrenewal and differentiation according to their internal properties and independently of the surrounding cells. If this is the case, then the question is why introducing a relatively complex hybrid model and not considering a simpler model, for example a system of ordinary differential equations. First of all, we need to note that this should be a cell population structured with respect to the intracellular drug concentration. Therefore, it should be a transport equation with time dependent coefficients and not ordinary differential equations. Second, since we introduce stochasticity in the problem, we need to study stochastic transport equations, which are not simpler than hybrid models. They do not admit analytical solution and should be solved numerically. Let us finally note that in a more complete model we would need to take into account both normal and leukemic cells, which compete for space. In this case, introduction of space distributions would be indispensable.

The hybrid model aims at describing realistically all levels of erythropoiesis. As more aspects of erythropoiesis get considered, such as global erythropoietin regulation or regulation of BFU-E and CFU-E from haematopoietic stem cells, we believe the hybrid model will be prove useful for designing biological experiments and guiding clinical practice.

Acknowledgments. We would like to thank the members of the INRIA projectteam Dracula for useful discussions. The work was partially supported by the ANR projects Mecamerge, Anatools and Bimod.

\section{REFERENCES}

[1] A. Altinok, F. Lévi And A. Goldbeter, Identifying mechanisms of chronotolerance and chronoefficacy for the anticancer drugs 5-fluorouracil and oxaliplatin by computational modeling, Eur. J. Pharm Sci., 36 (2009), pp. 20-38.

[2] A. Altinok, F. LÉvi And A. Goldbeter, A cell cycle automaton model for probing circadian patterns of anticancer drug delivery, Adv. Drug Deliv. Rev., 59 (2007), pp. 1036-1053. 
[3] A. Ballesta, S. Dulong, C. Abbara, B. Cohen, A. Okyar, J. Clairambault, F. Lévi, A Combined Experimental and Mathematical Approach for Molecular-based Optimization of Irinotecan Circadian Delivery, in press.

[4] S. Bernard, B. Cajavec Bernard, F. LÉvi and H. Herzel, Tumor growth rate determines the timing of optimal chronomodulated treatment schedules, PLoS Comput Biol., 6 (2010), e1000712.

[5] N. Bessonov, F. Crauste, I. Demin, V. Volpert, Dynamics of erythroid progenitors and erythroleukemia, Math. Model. Nat. Phenom., 4 (2009), No. 3, pp. 210-232.

[6] N. Bessonov, I. Demin, P. Kurbatova, L. Pujo-Menjouet, V. Volpert, Multi-Agent Systems and Blood Cell Formation, In: Multi-Agent Systems - Modeling, Interactions, Simulations and Case Studies, ISBN 978-953-307-176-3, edited by F. Alkhateeb, E. Al Maghayreh, I. A. Doush. http://www.intechopen.com/articles/show/title/multi-agentsystems-and-blood-cell-formation

[7] N. Bessonov, I. Demin, L. Pujo-Menjouet, V. Volpert, A multi-agent model describing self-renewal or differentiation effect of blood cell population, Mathematical and computer modelling, 49 (2009), pp. 2116-2127.

[8] N. Bessonov, P. Kurbatova, V. Volpert, Dynamics of growing cell populations, CRM, preprint num. 931 for Mathematical biology, University of Barcelona, (2010).

[9] N. Bessonov, P. Kurbatova, V. Volpert, Particle dynamics modelling of cell populations, Proc. Conf. JANO, Mohhamadia, 2008. Math. Model. Nat. Phenom., 5 (2010), No. 7, pp. $42-47$.

[10] N. Bessonov, L. Pujo-Menjouet, V, Volpert, Cell modelling of hematopoiesis, Math. Model. Nat. Phenom., 1 (2006), No. 2, pp. 81-103.

[11] J.A. Chasis And N. Mohandas, Erythroblastic islands: niches for erythropoiesis, Blood, 112 (2008), pp. 470-478.

[12] R. Cheong, A. Bergmann, S. L. Werner, J. Regal, A. Hoffmann and A. Levchenko, Transient I $\mathrm{B}$ Kinase Activity Mediates Temporal NF- $\kappa B$ Dynamics in Response to a Wide Range of Tumor Necrosis Factor- $\alpha$ Doses, The Journal of Biological Chemistry, 281 (2006), No. 5, pp. 2945-2950.

[13] L. Cojocaru and Z. Agur, A theoretical analysis of interval drug dosing for cell-cycle-phasespecific drugs, Mathematical biosciences, 109 (1992), No. 1, pp. 85-97.

[14] C. Colijn and M.C. Mackey, A mathematical model of hematopoiesis - I. Periodic chronic myelogenous leukemia, J. Theor. Biol., 237 (2005), pp. 117-132.

[15] C. Colijn And M.C. Mackey, A mathematical model of hematopoiesis - II. Cyclical neutropenia, J. Theor. Biol., 237 (2005), pp. 133-146.

[16] F. Crauste, I. Demin, O. Gandrillon, V. Volpert, Mathematical study of feedback control roles and relevance in stress erythropoiesis, J Theor Biol., 263 (2010), pp. 303-316.

[17] J.C. Dallon, Models with lattice-free center-based cells interacting with continuum environment variables, (2007), pp. 197-219.

[18] S. Dazy, F. Damiola, N. Parisey, H. Beug and O. Gandrillon, The MEK-1/ERKs signaling pathway is differentially involved in the self-renewal of early and late avian erythroid progenitor cells, Oncogene, 22 (2003), pp. 9205-9216.

[19] R. De Maria, U. Testa, L. Luchetti, A. Zeuner, G. Stassi, E. Pelosi, R. Riccioni, N. Felli, P. Samoggia and C. Peschle, Apoptotic Role of Fas/Fas Ligand System in the Regulation of Erythropoiesis, Blood, 93 (1999), pp. 796-803.

[20] I. Demin, F. Crauste, O. Gandrillon and V. Volpert, A multi-scale model of erythropoiesis, Journal of Biological Dynamics, 4 (2010), pp. 59-70.

[21] B.F. Dibrov, Resonance effect in self-renewing tissues, J. Theor. Biol., 192 (1998), pp. 15-33.

[22] D. Drasdo, Center-based single-cell models: An approach to multi-cellular organization based on a conceptual analogy to colloidal particles, Mathematics and Biosciences in Interaction (2007), pp. 171-196.

[23] E.H. Estey, Treatment of acute myeloid leukemia, Haematologica, 94 (2009), 10-16.

[24] S. Giacchetti, G. Buarnason, C. Garufi, D. Genet, S. Iacobelli, M. Tampellini, R. Smaaland, C. Focan, B. Coudert, Y. Humblet, J.L. Canon, A. Adenis, G. Lo Re, C. Carvalho, J. Schueller, N. Anciaux, M.A. Lentz, B. Baron, T. Gorlia, F. LÉVI, Phase III trial comparing 4-day chronomodulated therapy versus 2-day conventional delivery of fluorouracil, leucovorin, and oxaliplatin as first-line chemotherapy of metastatic colorectal cancer: the European Organisation for Research and Treatment of Cancer Chronotherapy Group, J. Clin. Oncol. 24 (2006), no. 22, pp. 3562-9.

[25] D.F. Gluzman, V.A. Nadgornaya, L.M. Sklyarenko, T.S. Ivanovskaya, L.Yu. PoludNENKO, N.I. UKRAINSKAYA, Immunocytochemical markers in acute leukemias diagnosis, Exp Oncol 2010 
[26] H.P. KoefFler, Induction of differentiation of human acute myelogenous leukemia cells: therapeutic implications, Blood, 62 (1983), no. 4, pp. 709-721.

[27] A. Kowal-Vern, F.M. Mazzella, J.D. Cotelingam, M.A. Shrit, J.T. Rector and H.R. SCHUMACHeR, Diagnosis and characterization of acute erythroleukemia subsets by determining the percentages of myeloblasts and proerythroblasts in 69 cases, Am. J. Hematol., 65 (2000), pp. 5-13.

[28] M. J. Koury AND M.C. Bondurant, Erythropoietin retards DNA breakdown and prevents programmed death in erythroid progenitor cells, Science, 248 (1990), pp. 378-381.

[29] A. Madan, C. Lin, Z. Wang and P.T. Curtin, Autocrine stimulation by erythropoietin in transgenic mice results in erythroid proliferation without neoplastic transformation, Blood Cells, Molecules and Diseases, 30 (2003), pp. 82-89.

[30] F.M. Mazzella, C. Alvares, A. Kowal-Vern and H.R. Schumacher, The acute erythroleukemias, Clin. Lab. Med., 20 (2000), pp. 119-37.

[31] R.L. Momparler, A Model for the Chemotherapy of Acute Leukemia with 1- $\beta$-D-Arabinofuranosylcytosine, Cancer Research, 34 (1974), pp. 1775-1787.

[32] M.C. Mormont And F. Levi, Cancer chronotherapy: principles, applications, and perspectives, Cancer., 97 (2003), pp. 155-69.

[33] P.F. Morrison, T.L. Lincoln And J. Aroesty, The disposition of ara-c and its metabolites: a pharmacokinetic simulation, Cancer Chemother. Rep., 59 (1975), pp. 861-876.

[34] J.M. Osborne, A. Walter, S.K. Kershaw, G.R. Mirams, A.G. Fletcher, P. Pathmanathan, D. Gavaghan, O.E. Jensen, P.K. Maini and H.M. Byrne, A hybrid approach to multi-scale modelling of cancer, Phil. Trans. R. Soc. A, 368 (2010), pp. 5013-5028.

[35] A.A. Patel, E.T. Gawlinsky, S.K. Lemieux and R.A. Gatenby, A Cellular Automaton Model of Early Tumor Growth and Invasion: The Effects of Native Tissue Vascularity and Increased Anaerobic Tumor Metabolism, J. Theor. Biol., 213 (2001), pp. 315-331.

[36] D.A. Rew, G.D. Wilson, I. Taylor and P.C. Weaver, Proliferation characteristics of human colorectal carcinomas measured in vivo, Br J Surg., 78 (1991), pp. 60-6.

[37] C. Rubiolo, D. Piazzolla, K. Meissl, H. Beug, J.C. Huber, A. Kolbus and M. Baccarini, A balance between Raf-1 and Fas expression sets the pace of erythroid differentiation, Blood, 108 (2006), pp. 152-159.

[38] R. Smaaland, O.D. Laerum, K. Lote, O. Sletvold, R.B. Sothern and R. Buerknes, DNA synthesis in human bone marrow is circadian stage dependent, Blood, 77 (1991), pp. 2603-2611.

[39] R. Smanland, R.B. Sothern, O.D. Laerum and J.F. Abrahamsen, Rhythms in human bone marrow and blood cells, Chronobiol Int., 19 (2002), pp. 101-27.

[40] M.Y. Yang, J.G. Chang, P.M. Lin, K.P. Tang, Y.H. Chen, H.Y. Lin, T.C. Liu, H.H. HsiaO, Y.C. LiU AND S.F. Lin, Downregulation of circadian clock genes in chronic myeloid leukemia: alternative methylation pattern of hPER3, Cancer Sci., 97 (2006), pp. 1298-1307.

[41] M.Y. YAnG, W.C. YAng, P.M. Lin, J.F. Hsu, H.H. HsiaO, Y.C. LiU, H.J. Tsai, C.S. Chang, S.F. Lin, Altered expression of circadian clock genes in human chronic myeloid leukemia, J. Biol. Rhythms. 26 (2011), no. 2, pp. 136-48. 